\title{
Spatial negative priming in bilingualism
}

\author{
BARBARA TRECCANI \\ University of Edinburgh, Edinburgh, Scotland \\ and University of Padua, Padua, Italy \\ AND \\ Efrosyni Argyri, Antonella Sorace, and Sergio Della Sala \\ University of Edinburgh, Edinburgh, Scotland
}

\begin{abstract}
Balanced bilinguals have been shown to have an enhanced ability to inhibit distracting information. In this study, we investigated the hypothesis that the bilinguals' efficiency in inhibitory control can be advantageous in some conditions, but disadvantageous in others - for example, negative priming conditions, in which previously irrelevant information becomes relevant. Data collected in a target-stimulus locating task from 29 early bilingual adults and 29 age-matched monolinguals showed that the bilinguals' greater inhibition of irrelevant spatial information (i.e., the position of a distractor stimulus) resulted in a smaller effect of the distractor presence (i.e., a smaller difference in error rates in trials with and without distractors) and a larger negative priming effect (i.e., a larger difference between the error rates shown in trials wherein the target position corresponded to the position of the previous-trial distractor and trials wherein the target was presented in a previously vacant position). These findings support the hypothesis of specific nonlinguistic cognitive effects of bilingualism on inhibitory control functions, which are not necessarily reflected in cognitive advantages.
\end{abstract}

One of the most intriguing questions about bilingualism is whether the continuous experience of handling two languages, and the mechanisms that bilinguals develop to control the two language systems, have any repercussions on nonlinguistic cognitive abilities. At least one model developed to account for the bilingual speaker's control over two simultaneously activated systems-Green's (1998) inhibitory control model - predicts that the management of two languages depends on the same general attentional resources used in nonverbal tasks.

Several studies reported on the advantages that bilingualism entails in nonlanguage domains. Earlier research on bilingual children (Bialystok, 1999; see Bialystok, 2001, for a review) obtained substantial evidence that bilingual children have an advantage in tasks that demand high levels of executive control. This advantage extends to nonverbal problem solving (Bialystok \& Martin, 2004) and numeracy (Bialystok \& Codd, 1997). Recent experiments on adult bilinguals led to the conclusion that the advantages of bilingualism persist well beyond childhood (Bialystok, 2006; Bialystok et al., 2005; Bialystok, Craik, Klein, \& Viswanathan, 2004; Bialystok, Craik, \& Ruocco, 2006; Bialystok, Craik, \& Ryan, 2006).

Most of the previous studies employed the Simon task (Simon \& Small, 1969) - that is, a choice reaction time (RT) task in which participants have to produce a lateralized response (e.g., a left- or right-sided keypress) on the basis of a nonspatial attribute of a lateralized stimulus (e.g., the color of a left- or right-sided stimulus). Bilinguals show a smaller Simon effect (i.e., the cost observed in trials in which the response and stimulus positions do not correspond as compared with that in trials in which they do correspond) than do monolinguals (Bialystok et al., 2004). This finding is assumed to reflect bilinguals' enhanced efficiency in inhibiting irrelevant information (i.e., the stimulus position in the Simon task), which would result from their massive practice in inhibiting one language when they speak the other.

However, a bilingual advantage in Simon tasks has been observed not only in spatially noncorresponding trials, but also in corresponding trials (Bialystok et al., 2004; Martin \& Bialystok, 2003). Similar results were obtained with other tasks that involve both conditions requiring the inhibition of misleading information and conditions in which no conflict should occur (see, e.g., Costa, Hernandez, \& Sebastián-Gallés, 2008). In order to account for this outcome, it has been proposed that bilingualism has a broad effect on cognitive abilities influencing not only inhibitory control functions, but also a wider range of cognitive processes. For example, Bialystok and her colleagues (Bialystok, 2006; Bialystok et al., 2004) maintained that bilingualism could also have an effect on working memory processes, such as those involved in holding in memory the relevant stimulus-response associations in Simon tasks (e.g., "press the left key when the stimulus is red and the right key when the stimulus is green").

B. Treccani, barbara.treccani@unipd.it 
Therefore, although differences between bilinguals and monolinguals have proved reliable, the bilinguals' advantage seems more general than initially hypothesized. One may then argue that the continuous experience of managing two languages results in nonspecific enhancement of cognitive resources that emerges when task demands are sufficiently high, rather than in effects on specific cognitive functions (see Norman \& Bobrow, 1975).

One straightforward way to evaluate whether bilingualism has a generic effect on the amount of resources available to perform cognitive tasks, or whether it has a selective effect on a specific cognitive function, is to find a condition in which a greater efficiency in exerting this function does not result in an advantage, but a disadvantage. Following this rationale, we employed a negative priming (NP) paradigm (Tipper, 1985) to test the hypothesis of bilinguals' greater efficiency in inhibitory control. NP is observed when the irrelevant information in one (prime) trial becomes relevant in the following (probe) trial. Slower and/or less accurate responses are usually produced in this condition, as opposed to a control condition in which neither the relevant nor the irrelevant information of the prime trial is presented in the probe trial. The NP effect is thought to reflect inhibition of the irrelevant information that exerts its effect from one trial to the next, and the magnitude of the NP effect seems to be connected with the strength of the inhibition. Indeed, NP may be reduced in people with deficits in inhibitory abilities, as revealed by their impaired performances in tasks that require inhibition of irrelevant information, such as patients affected by Alzheimer's disease (see Amieva, Phillips, Della Sala, \& Henry, 2004). If bilinguals are really more efficient than monolinguals at inhibitory control, as suggested by previous studies based on Simon paradigms (see, e.g., Bialystok et al., 2004), they should show a greater cost in critical NP conditions.

We used a spatial version of the NP paradigm (Tipper, Brehaut, \& Driver, 1990) — that is, a task in which the distracting information was represented by the position of an irrelevant stimulus that accompanied the target stimulus. In this task, participants were asked to respond by locating a target stimulus first in a prime display, and then again in a probe display. In both displays, four marked locations appeared, one of which was occupied by the target. In all the prime displays, the target was accompanied by a distractor (which occupied one of the other three locations and had to be ignored), whereas in probe displays, the target could be either accompanied or not by the distractor. This task allowed us to compare bilingual and monolingual performances on two different measures of inhibitory control efficiency. The prediction was that a greater ability at inhibiting distracting information (i.e., the distractor position) would result in a smaller effect of the distractor presence, and thus in a smaller difference between conditions in which only the target was presented and those in which the target was accompanied by the distractor. We also expected greater efficiency at inhibiting the irrelevant distractor position to result in a larger NP effect, and therefore in a larger difference between trials in which the target position corresponded to the position of the distractor presented in the previous trial, and trials in which the target and preceding-distractor positions did not correspond.

In contrast with previous studies that investigated bilinguals who were exposed to the second language after the age of 6 years (e.g., Bialystok et al., 2004), we tested balanced bilinguals who had been exposed to both languages from birth or from very early infancy.

\section{METHOD}

\section{Participants}

Twenty-nine bilingual and 29 monolingual adults participated in the experiment. All participants took a proficiency test in each of their languages and a nonverbal reasoning test (Raven's Standard Progressive Matrices [SPM]; Raven, 1938). The proficiency tests (score range 0-21) evaluated receptive vocabulary, knowledge of grammar, syntax, and reading comprehension, and were based on a placement test currently employed by the Greek language teaching center at the University of Athens. Versions of this test were created in each language spoken by the recruited participants to match the format and degree of difficulty of the Greek test. Bilingual participants also filled in a questionnaire about their previous and current language use, length and type of language exposure, and degree of fluency in their two languages. All of the included bilingual participants had been exposed to both languages within the first 6 years of life ( 25 participants had been exposed to both languages from birth, whereas for 3 participants the age of exposure to the second language was 4 years, and for 1 participant it was 6 years), had used these languages on a daily basis throughout their life, currently had an average use of either language ranging from $40 \%$ to $60 \%$, and had all obtained scores $\geq 18$ in the proficiency tests in both languages.

Among the bilingual participants, 26 were proficient in English and another European language (i.e., Greek, 20 participants; Italian, 4 participants; French, 1 participant), whereas 3 were bilingual in Greek and either Italian (2 participants) or French (1 participant). Seventeen bilingual participants had been living in the United Kingdom (U.K.) from birth; 3 bilinguals had been living in the U.K. for no less than 10 years and before had lived in Italy, South Africa, and Cyprus, respectively; 6 bilinguals had been living in the U.K. for no less than 5 years and had lived before in another European country (i.e., Greece, 4 participants; Italy, 1 participant; France, 1 participant); 2 bilinguals had been living in Greece for the last 4 and 6 years and had lived before in Belgium and Sweden, respectively; 1 bilingual had been living in Greece for the last 12 years and had lived in Italy before. The monolingual group consisted of 26 English-speaking and 3 Greek-speaking participants. These participants had been living from birth in the U.K. and in Greece, respectively. Monolingual participants were not functionally fluent in any other language; they all obtained a $\geq 18$ score on their own language proficiency test.

Sociodemographic characteristics and Raven's SPM scores of participants are shown in Table 1. The two groups did not significantly differ in age, years of education, or Raven's scores (all $p \mathrm{~s} \geq .50$ ). In addition, the two groups had the same or nearly the same compositions in terms of gender, handedness, achieved school degree, and occupation of participants.

\section{Stimuli, Apparatus, Design, and Procedure}

Each trial was initiated by pressing the space bar of the computer keyboard. Immediately afterward, four horizontal white lines that were $6.7^{\circ}$ long were drawn on the black background of the screen: two lines on the top and the other two on the bottom (see Figure 1). These lines remained on the screen throughout the trial and marked the locations at which the target and the possible distractor could appear. The middle points of the two top lines were $1.6^{\circ}$ above and $42.4^{\circ}$ to the left or right of the center of the screen, whereas those of the two bottom lines 
Table 1

Sociodemographic Characteristics and Raven's Standard Progressive Matrices Scores of Participants

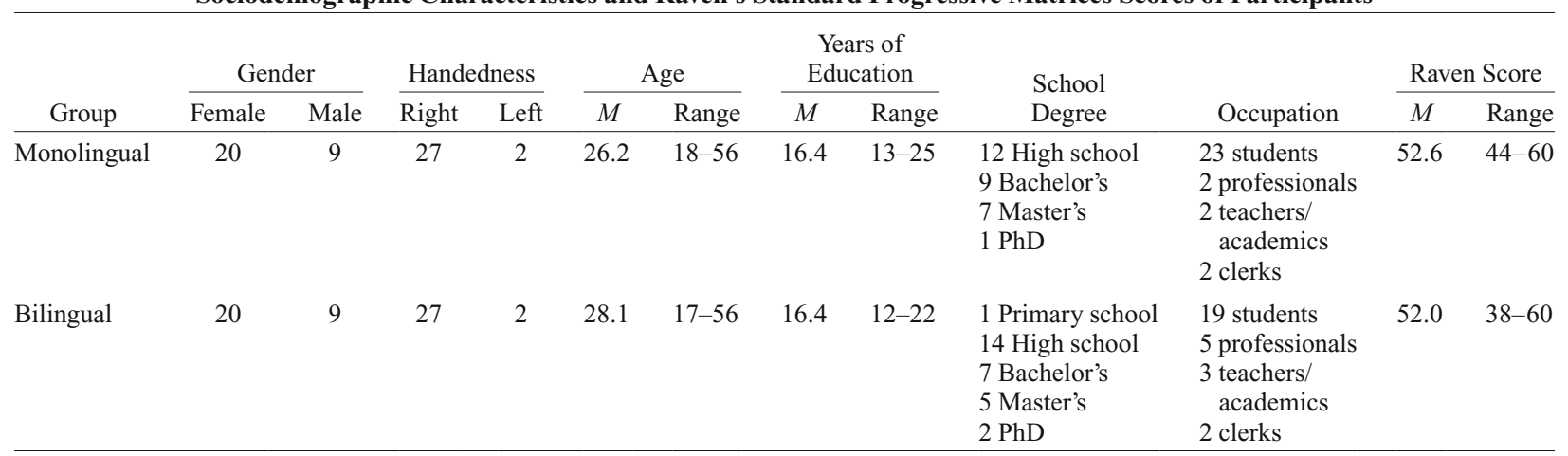

were $12.7^{\circ}$ below and $21.2^{\circ}$ to the left or right of the screen center. After 1,500 msec from the lines' onset, the prime display appeared for $150 \mathrm{msec}$ : A $6.0^{\circ} \times 9.2^{\circ} \mathrm{X}$ and $\mathrm{O}$ were drawn on the screen, each in one of the four marked locations. Responses were performed with the index and middle fingers of both hands and consisted of pressing one of four white-labeled keys ("d," "c," "k," and "m" characters on the computer keyboard), each spatially compatible with one of the four marked positions on the screen. Fifteen participants in both the monolingual and bilingual groups were asked to press the key the position of which corresponded to the $\mathrm{X}$ (i.e., the target) location and to ignore the $\mathrm{O}$ (i.e., the distractor) location, whereas the opposite mapping was assigned to the other participants. After either the response was made or the time granted for responding elapsed (3,000 msec after the prime's onset), there was a 350-msec interval (during which two different auditory feedbacks were given in case of either correct or wrong keypresses) before the probe display appeared. This display could exhibit either the target and the distractor or just the target. Again, the probe display remained visible for $150 \mathrm{msec}$; a maximum of 3,000 msec from the probe's onset was given to respond, and a 350-msec interval (during which the feedback was delivered) concluded the trial. The screen was then cleared, and a prompt to begin the next trial was presented.

Prime displays resulted from the combination of each one of the four locations as target position, with another of the remaining three locations as distractor position (12 possible combinations). The possible probe displays were 16 (12 target + distractor and 4 only-target displays). A particular display was never presented as both prime and probe in a given trial, and the probe distractor never occupied the location of the prime target. On the whole, there were 144 possible

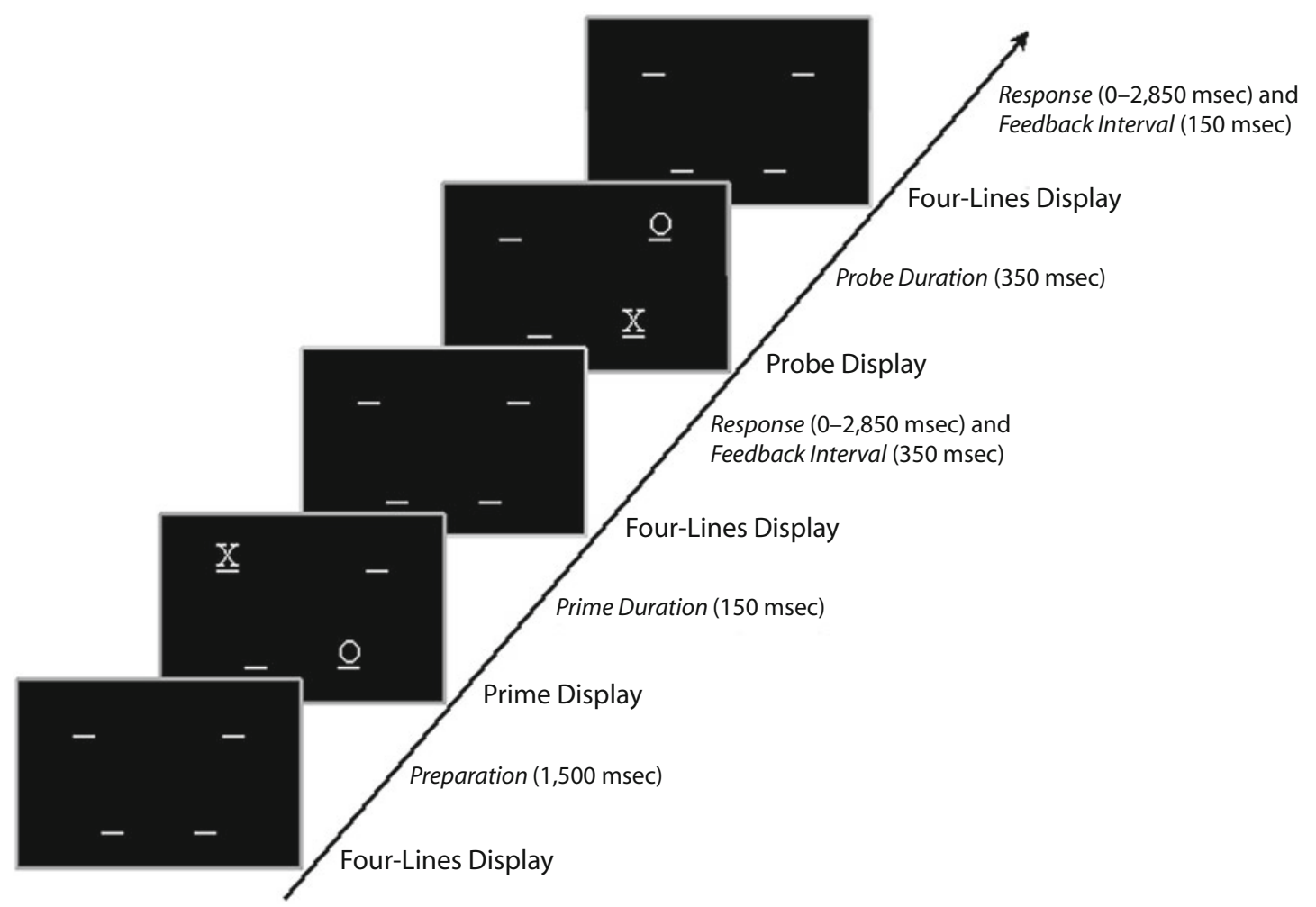

Figure 1. Diagram of the stimulus displays and timing of events in the spatial negative priming task. The prime displays consisted of four lines and two letters $(X$ and $O$ ). The probe displays consisted of the four lines and either both letters (as in the prime displays) or just one letter. See the text for further details. 


\section{Prime Display}

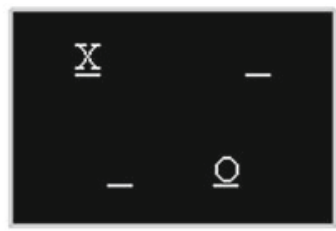

Probe Display

Target+Distractor/Unrelated Conditions
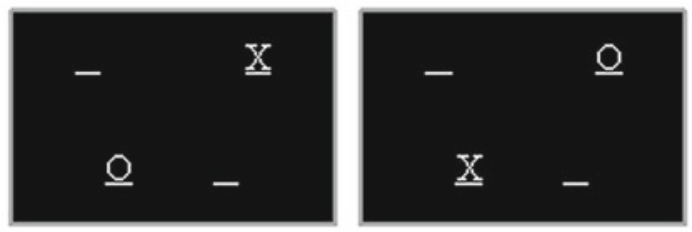

Only-Target/Unrelated Conditions
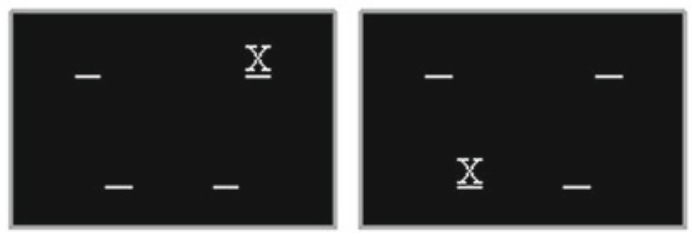

Target + Distractor/D $\rightarrow$ T Conditions
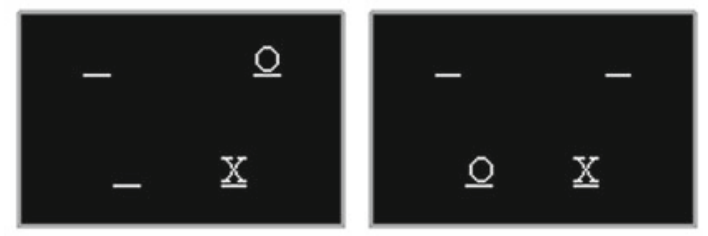

Only-Target/D $\rightarrow$ T Conditions

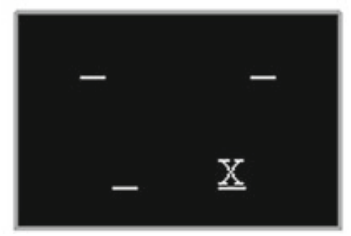

Figure 2. Examples of the prime display and possible probe displays in the four critical prime-probe conditions of the spatial negative priming task. In these examples, the target was $X$, whereas $O$ was the distractor. Probe displays on the top consist of the target and the distractor; in those on the bottom, no distractor is presented. In the probe displays on the left, the target is presented in a previously vacant position. In the displays on the right, the target position corresponds with that of the prime distractor.

prime-probe combinations. Each combination was presented once, and the 144 prime-probe trials were presented randomly in two blocks of 72 trials. Such a design results in equal probabilities concerning the location in which both the prime and the probe targets appeared; thus, there was no means for participants to predict the position of the probe target from the prime display. Critical conditions were those in which both the probe target and distractor appeared in previously vacant locations (i.e., target + distractor/unrelated conditions; 24 trials), those in which the probe target appeared in a previously vacant location and no distractor was presented in the probe display (i.e., only-target/unrelated conditions; 24 trials), and those in which the probe target appeared in the previous distractor locationeither when a probe distractor appeared in a previously vacant position (i.e., target + distractor/ $\mathrm{D} \rightarrow \mathrm{T}$ conditions; 24 trials) or no probe distractor was presented (i.e., only-target/D $\rightarrow T$ conditions; 12 trials). Examples of these four conditions are shown in Figure 2.

Other possible conditions were those in which the prime distractor location was repeated in the probe display and the probe target was presented in a previously vacant location (i.e., target + distractor/ $\mathrm{D} \rightarrow \mathrm{D}$ conditions; 24 trials) and those in which the prime target location was repeated in the probe display-either when a probe distractor appeared in a previously vacant location (i.e., target + distractor/ $\mathrm{T} \rightarrow \mathrm{T}$ conditions; 24 trials), or when no distractor was presented in the probe display (i.e., only-target/T $\rightarrow \mathrm{T}$ conditions; 12 trials).

\section{RESULTS}

Table 2 shows mean RTs and error percentages for the probe trials as a function of the distractor presence and prime-probe $(\mathrm{P}-\mathrm{P})$ location relationship.
Planned comparisons were performed on the data from the four critical conditions to test possible differences in the distractor and NP effects between the two groups. Findings from previous studies suggest that the appearance of the NP effect or its size may depend on whether the relevant information in the probe display is accompanied by distracting information, and, conversely, that the magnitude of the distractor effect may be affected by the P-P location relationship (see, e.g., Buckolz, Boulougouris, \& Khan, 2002; Tipper et al., 1990; Tipper \& Cranston, 1985). Accordingly, between-groups differences in the effect of each of the two relevant factors (distractor presence and P-P location relationship) were tested for each level of the other factor. Furthermore, pairedsamples $t$ tests were performed to test the distractor and NP effects separately for bilingual and monolingual participants (see Table 3).

In order to evaluate the distractor effect, monolinguals' and bilinguals' RTs in target+distractor trials were contrasted with those in only-target trials, separately for the two critical P-P location relationship conditions (unrelated, $\mathrm{D} \rightarrow \mathrm{T})$. No significant interactive effects were found in either of the two contrasts [both $F \mathrm{~s}(1,56) \leq 1.84$, $p \mathrm{~s} \geq .18$ ]. Monolinguals and bilinguals showed significant distractor effects in both unrelated and $\mathrm{D} \rightarrow \mathrm{T}$ trials $($ all $p s<.0005)$, and the size of these effects did not significantly differ between the two groups. 
Table 2

Spatial Negative Priming Task: Mean Reaction Times (RTs, in Milliseconds) and Percentages of Errors (PEs) of the Two Groups of Participants As a Function of Distractor Presence and Prime-Probe Location Relationship

\begin{tabular}{ccccc}
\hline Group & Distractor Presence & Prime-Probe Relationship & $\mathrm{RT}$ & PE \\
\hline Monolingual & Target+distractor & Unrelated & 439 & 4.2 \\
& & $\mathrm{D} \rightarrow \mathrm{T}$ & 474 & 5.3 \\
& & $\mathrm{~T} \rightarrow \mathrm{T}$ & 465 & 1.6 \\
& \multirow{3}{*}{ Only target } & $\mathrm{D} \rightarrow \mathrm{D}$ & 426 & 3.4 \\
& & Unrelated & 410 & 2.0 \\
& & $\mathrm{D} \rightarrow \mathrm{T}$ & 448 & 4.9 \\
Bilingual & Target+distractor & $\mathrm{T} \rightarrow \mathrm{T}$ & 413 & 0.3 \\
& & $\mathrm{Unnrelated}$ & 453 & 2.3 \\
& & $\mathrm{D} \rightarrow \mathrm{T}$ & 483 & 6.8 \\
& & $\mathrm{~T} \rightarrow \mathrm{T}$ & 480 & 1.6 \\
& & $\mathrm{D} \rightarrow \mathrm{D}$ & 438 & 2.7 \\
& Only target & $\mathrm{Unnelated}$ & 414 & 2.2 \\
& & $\mathrm{D} \rightarrow \mathrm{T}$ & 460 & 5.5 \\
& & $\mathrm{~T} \rightarrow \mathrm{T}$ & 423 & 0.3 \\
\hline
\end{tabular}

Note-D, distractor; $\mathrm{T}$, target.

The same analyses were performed on percentages of errors. A significant distractor presence $\times$ group interactive effect was found in the contrast that involved the unrelated trials $[F(1,56)=4.71, p<.05]$. As shown in Table 3 and Figure 3 , when the probe target was presented in a different location from that of the prime distractor, for bilinguals there were no differences between the two distractor presence conditions; that is, the distractor effect was absent $(p=.80)$. In contrast, monolinguals made more errors in target + distractor trials than in only-target trials $(p<.01)$. No significant effect was found in the contrast performed on $\mathrm{D} \rightarrow \mathrm{T}$ trials $[F(1,56)=0.21, p=$ .65]. Indeed, in these trials, neither monolingual nor bilingual participants showed significant distractor effects (both $p \mathrm{~s} \geq .43$ ).

To compare NP effects in the two groups, monolinguals' and bilinguals' RTs in $\mathrm{D} \rightarrow \mathrm{T}$ trials were contrasted with those in unrelated trials, separately for the two distractor presence conditions (absent, present). Neither contrast yielded significant effects [both $F \mathrm{~s}(1,56) \leq 0.97, p \mathrm{~s} \geq$ .33]. Monolingual and bilingual participants showed significant NP effects both when a probe distractor was presented and when no distractor was presented in the probe display (all $p \mathrm{~s}<.0001$ ), and the effects shown by the two groups did not significantly differ in size.

Percentages of errors underwent the same analyses that were performed on RTs. A significant interactive effect between P-P relationship and group was found in the contrast that involved target + distractor trials $[F(1,56)=$ $6.41, p<.05]$. When a distractor was presented in the probe display, monolinguals did not show significantly different levels of accuracy in the two P-P relationship conditions $(p=.10)$, whereas bilinguals produced more errors in $\mathrm{D} \rightarrow \mathrm{T}$ than in unrelated trials $(p<.0005$; see Table 3 and Figure 3 ). The contrast involving errors made in only-target trials proved to be nonsignificant $[F(1,56)=$ $0.05, p=.82]$. When no distractor was presented in the probe display, both monolingual and bilingual participants showed significant NP effects (both $p$ s $<.05$ ).

Finally, interactive contrasts were performed to compare monolinguals' and bilinguals' response speed and accuracy in unrelated conditions with those observed in the other three conditions provided for by the task-that is, the conditions wherein the location of either the prime target or distractor was repeated in the probe display (i.e., target+distractor/unrelated vs. both target+distractor/

Table 3

Spatial Negative Priming Task: Distractor Effects (Differences Between Target + Distractor and Only-Target Trials) in the Two Critical PrimeProbe Relationship Conditions, and Negative Priming (NP) Effects (Differences Between $\mathrm{D} \rightarrow \mathrm{T}$ and Unrelated Trials) in the Two Distractor Presence Conditions for Monolingual and Bilingual Participants, in Terms of Both Reaction Times (RTs, in Milliseconds) and Percentages of Errors (PEs)

\begin{tabular}{ccccccc}
\hline & & \multicolumn{2}{c}{ Distractor Effect } & & \multicolumn{2}{c}{ NP Effect } \\
\cline { 3 - 4 } \cline { 5 - 6 } Group & & Unrelated & $\mathrm{D} \rightarrow \mathrm{T}$ & & Target+Distractor & Only Target \\
\hline Monolingual & RT & $29^{* *}$ & $26^{* *}$ & & $35^{* *}$ & $38^{* *}$ \\
& PE & $2.2^{*}$ & 0.4 & & 1.1 & $2.9^{*}$ \\
\multirow{2}{*}{ Bilingual } & RT & $39^{* *}$ & $23^{* *}$ & & $30^{* *}$ & $46^{* *}$ \\
& PE & 0.1 & 1.3 & & $4.5^{* *}$ & $3.3^{*}$ \\
\hline
\end{tabular}

${ }^{*} p<.05 . \quad{ }^{* *} p<.0005$. 


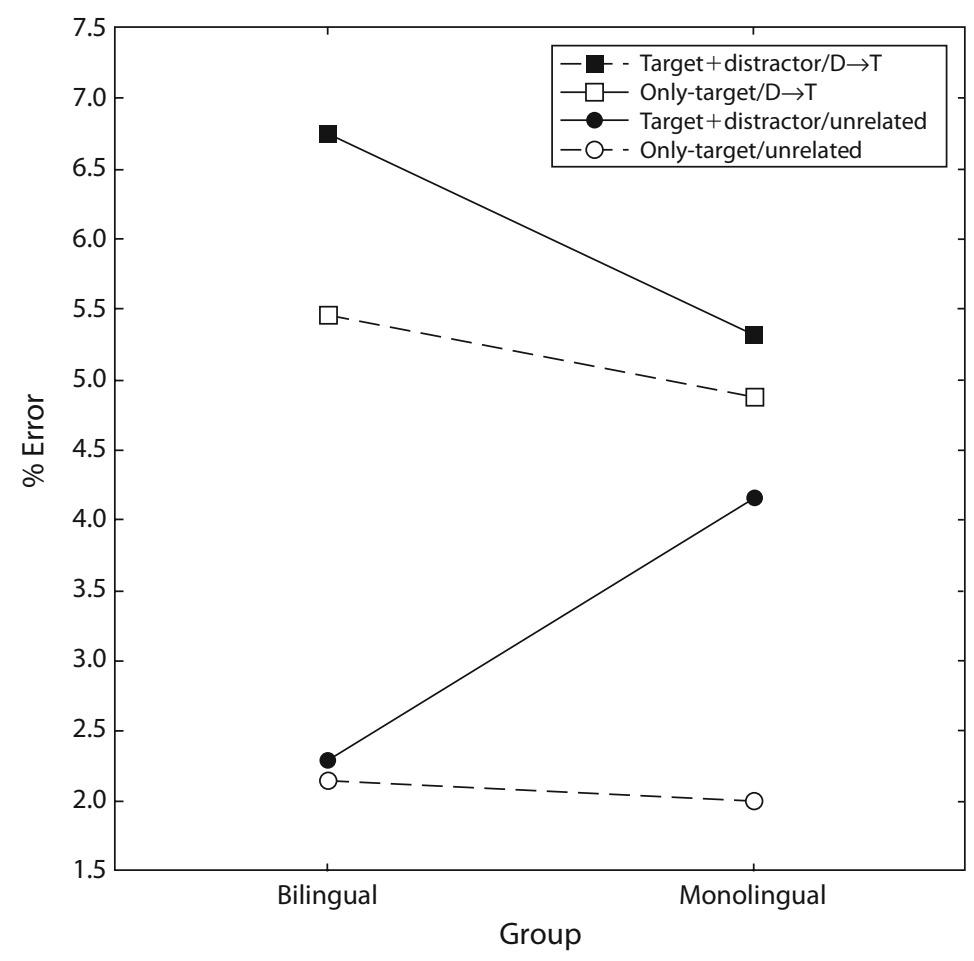

Figure 3. Percentages of errors of bilingual and monolingual participants in the four critical prime-probe conditions of the spatial negative priming task.

$\mathrm{T} \rightarrow \mathrm{T}$ and target + distractor/D $\rightarrow \mathrm{D}$; only-target/unrelated vs. only-target $/ T \rightarrow T)$. No significant effects were observed in both RT and error contrasts (all $p \mathrm{~s} \geq .15$ ).

\section{DISCUSSION}

In this study, a spatial NP paradigm that involved probe conditions both with and without distractor stimuli was administered to monolingual and bilingual participants. The dissociation between two measures of monolinguals' and bilinguals' performance was predicted on the basis of the supposed enhanced inhibitory control functions of bilinguals. On the one hand, bilingual participants were expected to show a smaller distractor effect than were monolinguals. This is consistent with prior studies that found a reduced Simon effect in bilinguals (e.g., Bialystok et al., 2004), which would reflect their enhanced ability to suppress misleading spatial information. In the present study, bilinguals' higher efficiency at inhibiting the position of the distractor should have resulted in a smaller cost of the distractor presence. On the other hand, NP was predicted to be larger in bilingual than in monolingual participants: The more efficient inhibition of the distractor position should have produced a larger cost when this position became relevant in the following trial. These predictions have been partially confirmed: The expected differences between the two groups of participants were observed in both the distractor effect and the NP effect, but only in response accuracy and in some of the tested conditions.

The difference between the accuracy shown in probe trials with and without a distractor was not significant for bilinguals in either of the two conditions of P-P location relationship, whereas monolinguals showed a significant distractor effect in the unrelated condition - that is, the condition in which the probe target was not presented in one of the previously primed positions. Indeed, this can be considered the optimal condition in which to test the effect of the distracting information in the present trial: In this condition, performance was not directly affected by the inhibitory processes triggered by the previous trial; thus, the distractor effect could not be masked by other effects, such as the NP effect. It is not surprising, therefore, that the predicted difference between bilinguals and monolinguals (i.e., a substantial distractor effect in monolinguals and the lack of the effect in bilinguals) was found only in this condition.

A converging pattern of results characterized the NP effect. Concerning response accuracy, bilinguals showed significant NP effects regardless of whether the probe target was accompanied by a distractor, whereas monolinguals exhibited a significant NP effect only when no probe distractor was presented. Therefore, the predicted difference between the two groups emerged in the target + distractor condition. In this condition, monolinguals made a relatively large percentage of errors even in unrelated trials, and the contribution of the NP to the performance was small and not significant. In contrast, bilinguals' performance was not dramatically affected by the distractor presence in unrelated trials, but their accuracy significantly worsened in $\mathrm{D} \rightarrow \mathrm{T}$ trials.

When RTs were taken into account, opposite trends (i.e., a numerically larger difference between trials with and 
without the distractor in bilinguals or a larger difference between unrelated and $\mathrm{D} \rightarrow \mathrm{T}$ trials in monolinguals) were observed in some of the tested conditions. Although the presence of these trends calls for a certain caution in drawing strong conclusions from the present results, it should be noticed that both monolinguals and bilinguals showed large and highly significant distractor and NP effects in terms of response speed, and that the small differences in magnitude between the RT effects of the two groups of participants were far from significant and likely to be meaningless.

No differences between the two groups were found when the unrelated conditions were contrasted with either repeated target- or distractor-location conditions. Bilingualism, therefore, seems to have had a selective effect on participants' performance. It specifically influenced the effect of irrelevant spatial information presence (i.e., the distractor effect) and that of the irrelevant information becoming relevant (i.e., the NP effect) on participants' accuracy. Furthermore, it is worth noticing that the NP task used in the present study made fewer demands on working memory than did Simon tasks, which require holding different sets of instructions in memory: Participants, in fact, had to remember only the identity of the target. Therefore, the bilingual-monolingual differences in the size of the two effects of irrelevant spatial information do not seem to be attributable to cognitive components other than inhibitory control functions. Moreover, the fact that we found differences in opposite directions between the two groups in the size of distractor and NP effects rules out an account of these differences in terms of unequal levels of cognitive resources. A similar conclusion could not be reached if only one effect (i.e., only one measure of inhibitory control) had been examined. The lack of a particular effect (e.g., the distractor effect) in one of the two groups of participants, and the presence of such an effect in the other group, could be due to a greater availability of cognitive resources in the former group (see Norman \& Bobrow, 1975; Shallice, 1988), which would allow these participants to execute the task in the difficult and easy conditions (e.g., the target+distractor and only-target conditions) at the same, or almost the same, level of performance. An explanation in terms of unequal availability of cognitive resources is unlikely if the two groups do not perform differently in a second measure (e.g., a task that is supposed to tap a cognitive function other than inhibitory control; see Bialystok, 2006), or if the opposite dissociation is observed in a second measure, as was the case in the present study - that is, if the difference between the performances observed in two other conditions (e.g., the $\mathrm{D} \rightarrow \mathrm{T}$ and unrelated conditions) is larger for the group of participants who did not show a significant effect when the first measure was taken into account.

In conclusion, our results represent evidence in support of a substantial effect of bilingualism on executive control processing. Bilingual and monolingual participants did not differ in overall response speed and accuracy. However, where significant differences between the two groups were observed, these differences were consistent in indicating that bilinguals are more efficient than monolinguals at inhibiting distracting information. Bilingual participants benefited from their higher efficiency when they had to locate a target stimulus while ignoring an irrelevant distractor: In trials where the target was not presented in a previously primed position, the interference effect of the distractor on response accuracy was smaller in bilinguals than in monolinguals. This enhanced efficiency, however, turned into a disadvantage in conditions in which the previously inhibited information became relevant for the task: When the probe target was accompanied by a distractor and was presented in the prime distractor position, the negative effect of the previously inhibited position on participants' accuracy was larger in bilinguals than in monolinguals. Crucially, therefore, the effect of bilingualism on executive control may translate into behavioral advantages or disadvantages, depending on the characteristics of the task.

\section{AUTHOR NOTE}

This research was supported by a grant from the Development Trust Research Fund of the University of Edinburgh awarded to A.S. and S.D.S. Address correspondence to B. Treccani, Human Cognitive Neuroscience, Psychology, University of Edinburgh, 7 George Square, Edinburgh EH8 9JZ, Scotland (e-mail: barbara.treccani@unipd.it).

\section{REFERENCES}

Amieva, H., Phillips, L. H., Della Sala, S., \& Henry, J. D. (2004) Inhibitory functioning in Alzheimer's disease. Brain, 127, 949-964.

BialystoK, E. (1999). Cognitive complexity and attentional control in the bilingual mind. Child Development, 70, 636-644.

BialystoK, E. (2001). Bilingualism in development: Language, literacy, and cognition. New York: Cambridge University Press.

BiALYSTOK, E. (2006). Effect of bilingualism and computer video game experience on the Simon task. Canadian Journal of Experimental Psychology, 60, 68-79.

Bialystok, E., \& CodD, J. (1997). Cardinal limits: Evidence from language awareness and bilingualism for developing concepts of number. Cognitive Development, 12, 85-106.

Bialystok, E., Craik, F. I. M., Grady, C., Chau, W., Ishit, R., Gunj, A., \& Pantev, C. (2005). Effect of bilingualism on cognitive control in the Simon task: Evidence from MEG. NeuroImage, 24, 40-49.

Bialystok, E., Craik, F. I. M., Klein, R., \& Viswanathan, M. (2004) Bilingualism, aging, and cognitive control: Evidence from the Simon task. Psychology \& Aging, 19, 290-303.

Bialystok, E., Craik, F. I. M., \& Ruocco, A. C. (2006). Dual-modality monitoring in a classification task: The effects of bilingualism and ageing. Quarterly Journal of Experimental Psychology, 59, 19681983.

Bialystok, E., Craik, F. I. M., \& Ryan, J. (2006). Executive control in a modified antisaccade task: Effects of aging and bilingualism. Journal of Experimental Psychology: Learning, Memory, \& Cognition, 6, 1341-1354.

Bialystok, E., \& Martin, M. M. (2004). Attention and inhibition in bilingual children: Evidence from the dimensional change card sort task. Developmental Science, 7, 325-339.

Buckolz, E., Boulougouris, A., \& Khan, M. (2002). Influence of probe-trial selection on the location negative priming effect. Canadian Journal of Experimental Psychology, 56, 273-282.

Costa, A., Hernandez, M., \& Sebastián-Gallés, N. (2008). Bilingualism aids conflict resolution: Evidence from the ANT task. Cognition, 106, 59-86

Green, D. W. (1998). Mental control of the bilingual lexico-semantic system. Bilingualism: Language \& Cognition, 1, 67-81.

Martin, M. M., \& BialystoK, E. (2003, October). The development of two kinds of inhibition in bilingual and monolingual children: Simon vs. Stroop. Poster session presented at the biennial meeting of the Cognitive Development Society, Park City, UT. 
Norman, D. A., \& Bobrow, D. G. (1975). On data-limited and resourcelimited processes. Cognitive Psychology, 7, 44-64.

Raven, J. C. (1938). Progressive matrices: A perceptual test of intelligence. London: H. K. Lewis.

Shallice, T. (1988). From neuropsychology to mental structure. Cambridge: Cambridge University Press.

Simon, J. R., \& Small, A. M. (1969). Processing auditory information: Interference from an irrelevant cue. Journal of Applied Psychology, 53, 433-435.

TIPPER, S. P. (1985). The negative priming effect: Inhibitory priming by ignored objects. Quarterly Journal of Experimental Psychology, 37A, 571-590.
TiPPER, S. P., BRehaUt, J. C., \& DrIVER, J. (1990). Selection of moving and static objects for the control of spatially directed action. Journal of Experimental Psychology: Human Perception \& Performance, 16, 492-504.

Tipper, S. P., \& CRAnston, M. (1985). Selective attention and priming: Inhibitory and facilitatory effects of ignored primes. Quarterly Journal of Experimental Psychology, 37A, 591-611.

(Manuscript received November 3, 2007; revision accepted for publication October 26, 2008.) 